\title{
3-Hydroxykynurenine and 3-Hydroxyanthranilic Acid Enhance the Toxicity Induced by Copper in Rat Astrocyte Culture
}

\author{
Daniela Ramírez-Ortega, ${ }^{1,2}$ Alelí Ramiro-Salazar, ${ }^{3}$ Dinora González-Esquivel, ${ }^{1}$ Camilo Ríos, ${ }^{1}$ \\ Benjamín Pineda, ${ }^{3}$ and Verónica Pérez de la Cruz ${ }^{1}$ \\ ${ }^{1}$ Departamento de Neuroquímica, Instituto Nacional de Neurología y Neurocirugía Manuel Velasco Suárez, S.S.A, \\ 14269 México City, MEX, Mexico \\ ${ }^{2}$ Biochemistry, Universidad Nacional Autónoma de México (UNAM), México City, MEX, Mexico \\ ${ }^{3}$ Laboratorio de Neuroinmunología, Instituto Nacional de Neurología y Neurocirugía Manuel Velasco Suárez, S.S.A, \\ 14269 México City, MEX, Mexico
}

Correspondence should be addressed to Benjamín Pineda; benpio76@hotmail.com and Verónica Pérez de la Cruz; veped@yahoo.com.mx

Received 24 March 2017; Accepted 11 June 2017; Published 31 July 2017

Academic Editor: Andrea Romani

Copyright ( 2017 Daniela Ramírez-Ortega et al. This is an open access article distributed under the Creative Commons Attribution License, which permits unrestricted use, distribution, and reproduction in any medium, provided the original work is properly cited.

\begin{abstract}
Copper is an integral component of various enzymes, necessary for mitochondrial respiration and other biological functions. Excess copper is related with neurodegenerative diseases as Alzheimer and is able to modify cellular redox environment, influencing its functions, signaling, and catabolic pathways. Tryptophan degradation through kynurenine pathway produces some metabolites with redox properties as 3-hydroxykynurenine (3-HK) and 3-hydroxyanthranilic acid (3-HANA). The imbalance in their production is related with some neuropathologies, where the common factors are oxidative stress, inflammation, and cell death. This study evaluated the effect of these kynurenines on the copper toxicity in astrocyte cultures. It assessed the $\mathrm{CuSO}_{4}$ effect, alone and in combination with 3-HK or 3-HANA on MTT reduction, ROS production, mitochondrial membrane potential (MMP), GHS levels, and cell viability in primary cultured astrocytes. Also, the chelating copper effect of 3-HK and 3-HANA was evaluated. The results showed that CuSO4 decreased MTT reduction, MMP, and GSH levels while ROS production and cell death are increasing. Coincubation with 3-HK and 3-HANA enhances the toxic effect of copper in all the markers tested except in ROS production, which was abolished by these kynurenines. Data suggest that 3-HK and 3-HANA increased copper toxicity in an independent manner to ROS production.
\end{abstract}

\section{Introduction}

Metals have a vital participation in some cellular processes as enzyme cofactors, as structural and antioxidant components, and also as part of metabolism. For this reason, their balance in cell environment is important and their unbalance causes damage in elemental cell structures as lipids and DNA; therefore, metals excess can be toxic mainly by oxidative stress production [1-3]. Copper is the third most abundant essential transition metal that is naturally found in human liver [4] and the most abundant in the brain [5]; this metal works as structural and functional part in various systems. In CNS, copper can act as cofactor of some antioxidant enzymes like + copper/zinc-dependent superoxide dismutase (SOD-1) and has big importance in respiration mitochondrial respiration as part of cytochrome $c$ oxidase structure [6, 7]. In addition, this metal participates in the neurotransmitter biosynthesis (noradrenaline) and can be stored in ceruloplasmin [8-10]. Copper can be transported into the brain through copper transporter 1 (Ctr1) and toward inner of brain cells by the ATPase copper transporter (ATP7A) and the divalent metal transporter (DMT1) [11-15]. It has been reported that astrocytes have a great influence in cerebral copper homeostasis and they can store big amounts of this metal 
due to specific characteristics as vast amounts of DMT1, ferritin, metallothionein, and antioxidants as glutathione (GSH) $[13,16,17]$. However, alterations in copper metabolism have been related with neurodegenerative diseases as Alzheimer's (AD), Parkinson's (PD), Menkes (MD), and Wilson's (WD) diseases, triggering an oxidative stress state in cell environment, resulting in disturbance of energy metabolism and reactive oxygen species (ROS) production [18-21]. ROS can also stimulate endogenous pathways that can be modulated by redox environment as the kynurenine pathway (KP).

$\mathrm{KP}$ is the main route of tryptophan (Trp) catabolism. Trp is an essential amino acid, which can be metabolized through different pathways to form important substances as serotonin and melatonin, but more than $95 \%$ is degraded through KP [22], whose main aim is $\mathrm{NAD}^{+}$ production, an electron carrier and cofactor in some redox reactions [23]. KP is present in the liver, kidney, and brain of various mammals such as mice, rats, guinea pigs, rabbits, monkeys, and humans [22, 24, 25]. Along the pathway, different metabolites with neuroactive activity and/or redox properties are produced. KP is highly regulated by redox status of the cellular environment, but its metabolites can also modify this environment due to their redox properties [26]. The alteration in KP metabolite levels has been associated with aging and several neurodegenerative diseases as Huntington, Parkinson, and Alzheimer [27]. Specifically, 3-hydroxykynurenine (3-HK) and 3-hydroxyanthranilic acid (3-HANA) have been studied by various research groups, which describe controversial results. The metabolite $3-\mathrm{HK}$ is found at nanomolar concentrations in CNS in normal conditions, but its levels are modified in neurodegenerative diseases. In fact, the amount increases as much as three times in Huntington's disease $[28,29]$. It has been reported that $3-\mathrm{HK}$ is able to induce cell death through apoptosis in brain regions as well as in cell cultures and in vivo experiments (with DNA fragmentation and chromatin condensation) [30-33]. In addition, it has been shown that $3-\mathrm{HK}$ generates oxidative stress besides that it triggers protein aggregates in human lens and finally cataract formation because of its interaction with metals [33-35]. On the other hand, there are reports where it was observed that $3-\mathrm{HK}(0-100 \mu \mathrm{M})$ works as an antioxidant. In this context, 3-HK and 3-HANA were able to decrease lipid peroxidation and GSH oxidation in brain cortex homogenates [36]; in Aldrichina grahami homogenates, 3$\mathrm{HK}$ was able to trap superoxide [37]. In other reports, it has been demonstrated that $3-\mathrm{HK}$ can capture hydroxyl and peroxyl radicals $[36,38]$. Recently, it was shown that $3-\mathrm{HK}$ can have chelating properties with metals as ferrous and also can scavenge $\mathrm{OH} \bullet$ and $\mathrm{ONOO}^{-}$in chemical combinatory assays [39].

Moreover, 3-HANA has also ambiguous characteristics which cause toxicity in neuronal cultures and can produce protein damage due to its interaction with metals and with the ability to generate hydroxyl radicals through Fenton's reaction. Besides, it has been reported that 3-HANA can have uncoupling effect in oxidative phosphorylation and is able to decrease oxygen consumption-activating astrocytes and neuron death [33, 34, 40-42]. Nevertheless, 3-HANA is described as scavenger of $\mathrm{OH} \bullet$ and $\mathrm{ONOO}^{-}$in chemical combinatory assays and can act as a chelator of ferrous ion $[36,39]$. In addition, 3-HANA can be an inflammatory and neuroprotector molecule since it induces hemeoxygenase-1 and suppresses cytokine and chemokine production stimulated by IL-1/IFN- $\gamma$ and toll-like receptor (TLR) ligands leading to neuroprotection [43].

Due to the fact that $3-\mathrm{HK}$ and 3-HANA influence the redox environment and knowing that copper can be toxic to the cell, the aim of this work was to determinate the effect of the coincubation of copper with these two kynurenine metabolites, in the toxicity induced by this metal.

\section{Materials and Methods}

3-hydroxykynurenine (3-HK), 3-hydroxyanthranilic acid (3HANA), copper sulfate $\left(\mathrm{CuSO}_{4}\right)$, thiazolyl blue tetrazolium bromide, 2',7'-diclorodihidrofluoresceine diacetate (DCFDA), and propidium iodide (PI) were obtained from Sigma Chemical Company (St. Louis, MO, USA). Dulbecco's modified eagle's medium (DMEM) and fetal bovine serum (FBS) were purchased from Gibco BRL (Grand Island, NY). All other chemicals were of the highest commercially available purity and obtained from known commercial suppliers. Solutions were prepared using deionized water obtained from a Milli-RQ (Millipore) purifier system.

2.1. Copper Chelation Assays. Chelation capacity of both KP metabolites was assessed according to previous report [44], where different concentrations of $3-\mathrm{HK}$ and 3-HANA (0-1 mM) were tested. Briefly, a solution of chelator $(50 \mu \mathrm{l}$ of 3-HK or 3-HANA in different concentrations) was mixed with $\mathrm{CuSO}_{4}(50 \mu \mathrm{l})$ in HEPES buffer $(50 \mu \mathrm{l})$. After 2 minutes, $50 \mu$ l of hematoxylin or DMSO (blank) was added and mixed for 3 minutes. Then, the absorbance was measured during $4 \mathrm{~min}$. The wavelength used was different for each $\mathrm{pH}$ tested. Three different $\mathrm{pH}(5.5,6.8$, and 7.5) and 2 different buffers were tested (sodium acetate buffer pH5.5 and HEPES buffer pH6.8 and 7.5), considering previous reports where it was demonstrated that copper accumulation, as in the pathologies, can change $\mathrm{pH}$ environment $[45,46]$.

2.2. Primary Astrocyte Cultures. Rat-cultured cortical astrocytes were obtained from the brains of 3 days postnatal Wistar rats (PND). Cells were seeded in Roux flasks at a $9 \times 10^{6}$ cells/ml density. The cells were maintained in DMEM supplemented with FBS at $10 \%$ under incubation at $37^{\circ} \mathrm{C}$ with $\mathrm{CO}_{2}(5 \%)$, until the cells were again seeded in 24-well plates to be used. Over 95\% of the cells were immunoreactive for glial fibrillary acidic protein, an astrocyte-specific marker [39].

2.3. MTT Reduction Assay. According to previous reports $[39,47,48]$, cellular function was evaluated by MTT reduction assay. This assay is employed as a functional status test through the formation of formazan salts by the action of dehydrogenases in viable cells [39]. Briefly, astrocytes (100,000 per well) were treated with different copper concentrations $(0-500 \mu \mathrm{M})$, to stablish the toxic copper concentration. Then, $\mathrm{CuSO}_{4}(350 \mu \mathrm{M})$ was coincubated with $3-\mathrm{HK}$ 


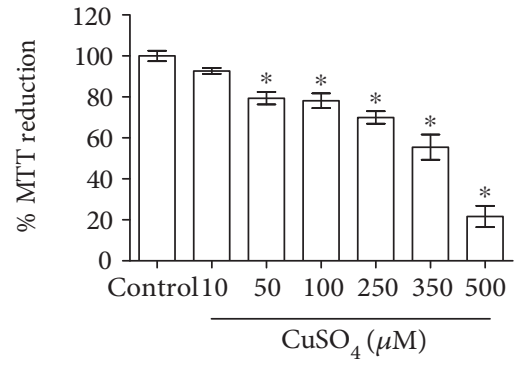

(a)

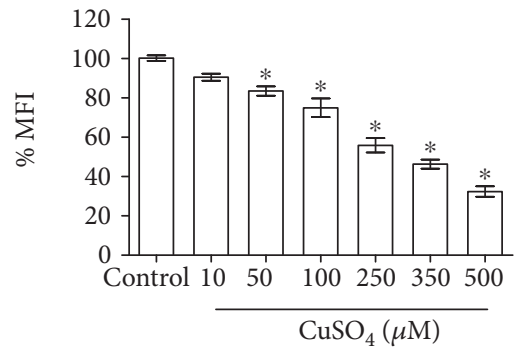

(c)

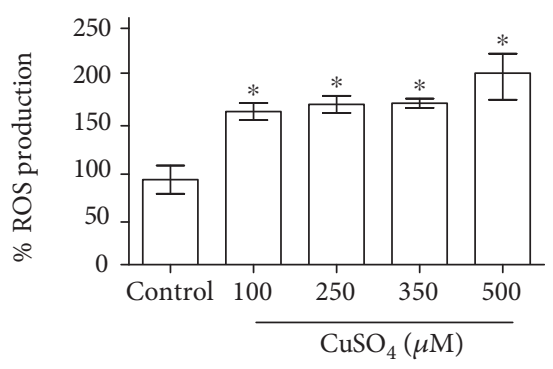

(b)

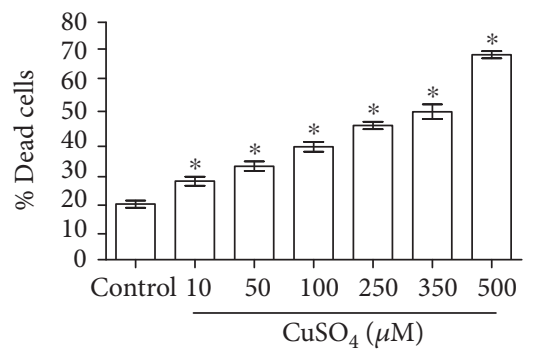

(d)

FIGURE 1: Effect of $\mathrm{CuSO}_{4}$ on cellular function (a), ROS production (b), MMP (c), and cell viability (d) in astrocytes. After incubation for $24 \mathrm{~h}$ with copper, MTT, DCFC-DA, JC-1, and iodide propidium were added to each well, respectively. Data are presented as mean values + SEM of 8 independent experiments from 4 different cultures. ${ }^{*} p<0.05$ versus control (one-way ANOVA followed by Tukey's test).

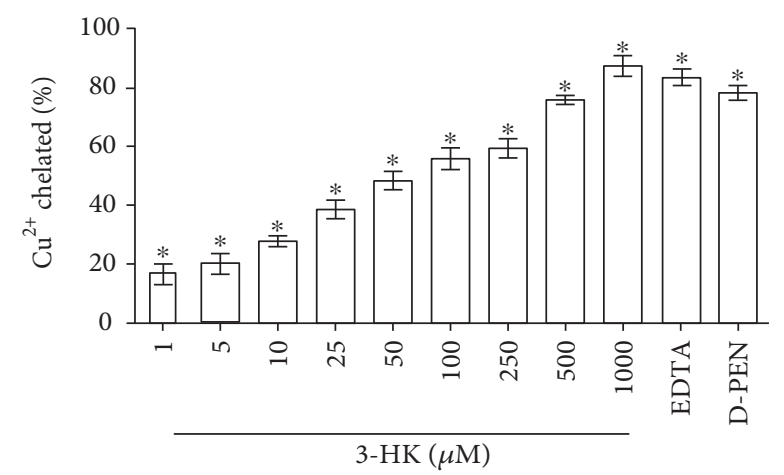

(a)

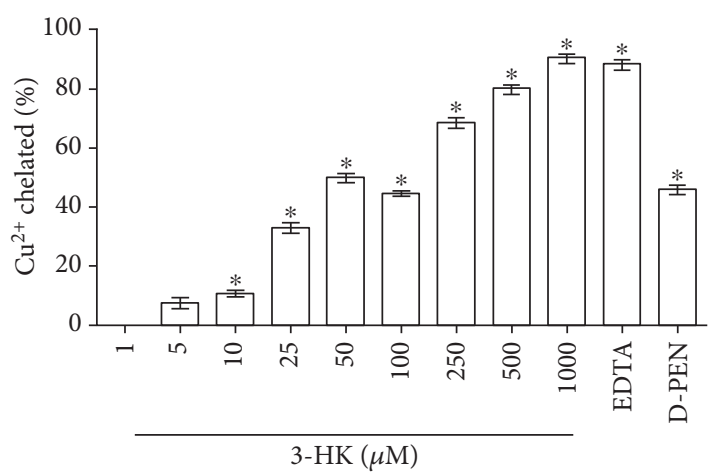

(b)

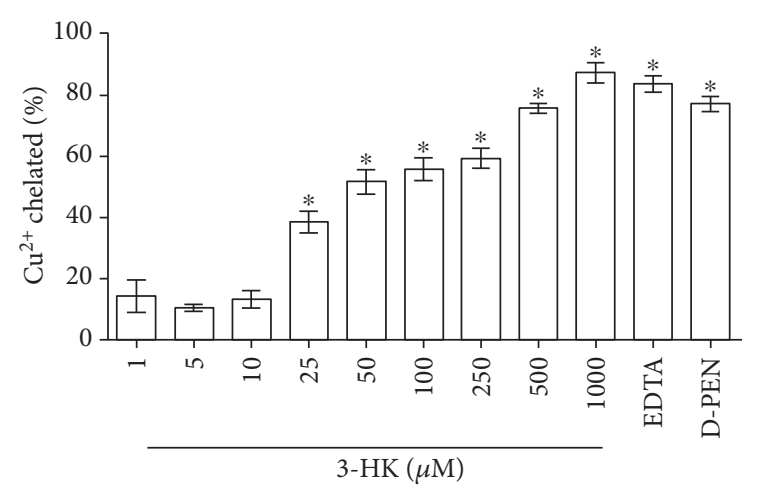

(c)

Figure 2: Copper chelation capacity of 3-HK. Different conditions of pH were tested: pH 5.5 (a), pH 6.8 (b), and pH 7.5 (c). Data are presented as mean values + SEM of 8 independent experiments for each concentration. ${ }^{*} p<0.001$ versus control (one-way ANOVA followed by Tukey's test). 


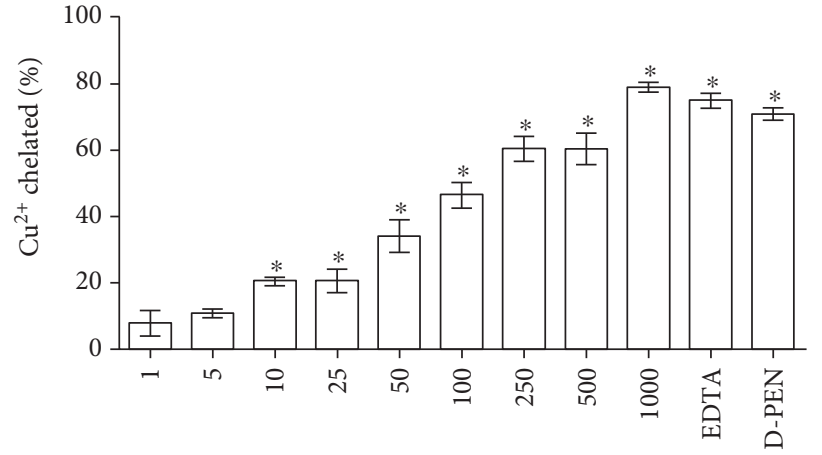

3-HANA $(\mu \mathrm{M})$

(a)

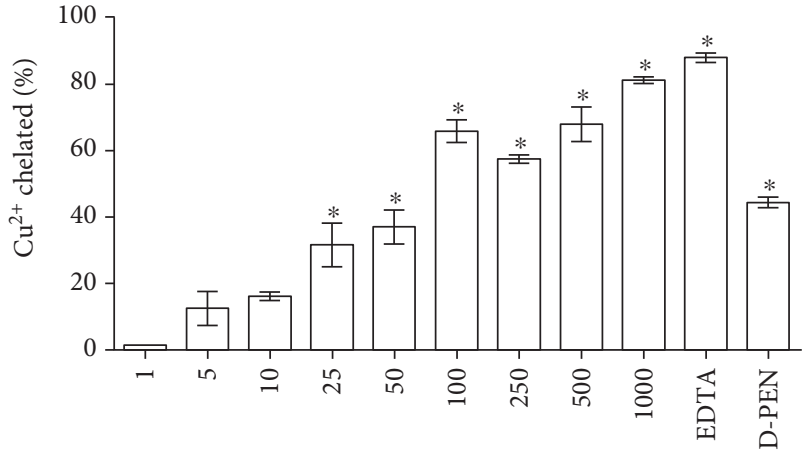

3-HANA $(\mu \mathrm{M})$

(b)

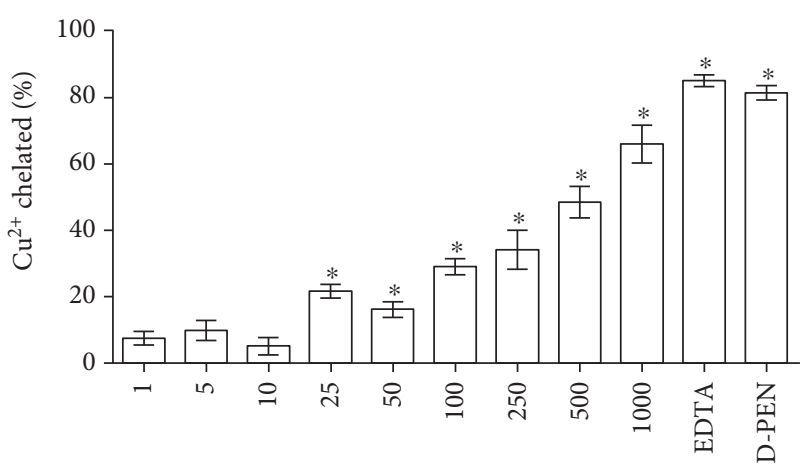

3-HANA $(\mu \mathrm{M})$

(c)

Figure 3: Copper chelation capacity of 3-HANA. Different conditions of pH were tested. pH 5.5 (a), pH 6.8 (b), and pH 7.5 (c). Data are presented as mean values + SEM of 8 independent experiments for each concentration. ${ }^{*} p<0.001$ versus control (one-way ANOVA followed by Tukey's test).

and 3-HANA $(100 \mu \mathrm{M})$ in DMEM medium for $24 \mathrm{~h}$ at $37^{\circ} \mathrm{C}$. After treatment, the medium was removed and $500 \mu \mathrm{l} \mathrm{MTT}$ $(1 \mathrm{mg} / \mathrm{ml}$ in DMEM medium) was added to each well. MTT was incubated for $3 \mathrm{~h}$ at $37^{\circ} \mathrm{C}$, then medium was removed, and acid isopropanol was added to dissolve the blue formazan salts. Quantification of resulting blue formazan salts was done at a wavelength of $570 \mathrm{~nm}$ in a plate reader (EON, BioTek). The results were expressed as the percentage of MTT reduction versus control values.

2.4. ROS Production Determination. ROS were evaluated through DCF-DA oxidation [49]. Astrocytes (100,000 per well) were treated with different copper concentrations (0$500 \mu \mathrm{M})$, and then copper $(350 \mu \mathrm{M})$ was coincubated with 3 -HK and 3-HANA $(100 \mu \mathrm{M})$ in DMEM medium for $24 \mathrm{~h}$ at $37^{\circ} \mathrm{C}$. After that, medium was removed, and cells were washed with saline solution and were added with $75 \mu \mathrm{l}$ of trypsin. Cells were recollected, and $100 \mu \mathrm{l}$ of DCF-DA $(75 \mu \mathrm{M})$ was added to the tubes and reincubated for $20 \mathrm{~min}$ at $37^{\circ} \mathrm{C}$ in darkness. After incubation, ROS formation was quantified by flow cytometry at $488 \mathrm{~nm}$ excitation and 532 emission considering 10,000 total events in FlowJo programm. Data are presented as percentage of ROS production versus control.

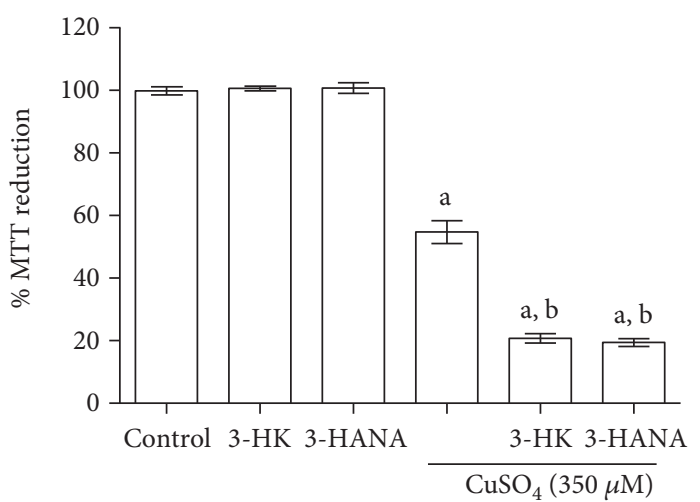

FIGURE 4: Effect of 3-HK and 3-HANA in the cellular dysfunction induced by $\mathrm{CuSO}_{4}(350 \mu \mathrm{M})$. After $24 \mathrm{~h}$ of incubation with 3 -HK or 3 -HANA $(100 \mu \mathrm{M})+$ copper, MTT was added to each well and formazan salt was measured. Data are presented as mean values + SEM of 6 independent experiments from 3 different cultures. ${ }^{\mathrm{a}} p<0.001$ versus control and ${ }^{\mathrm{b}} p<0.001$ versus $\mathrm{CuSO}_{4}$ (one-way ANOVA followed by Tukey's test).

2.5. Mitochondrial Membrane Potential (MMP) Assay. Mitochondrial membrane potential is a marker of healthy cells, and JC-1, a lipophilic cation, is used to evaluate because it is 


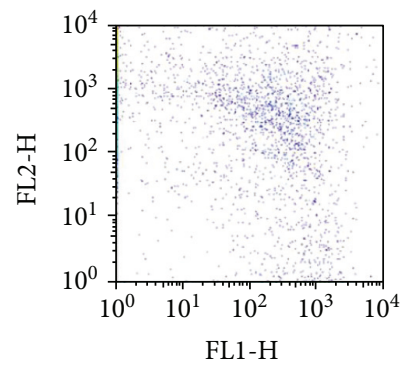

(a)

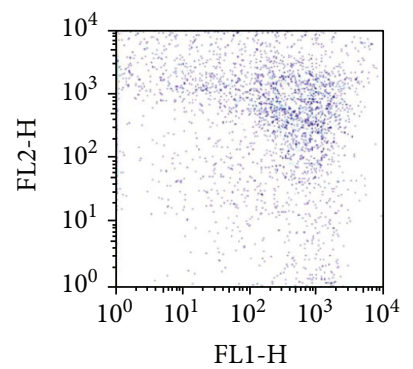

(d)

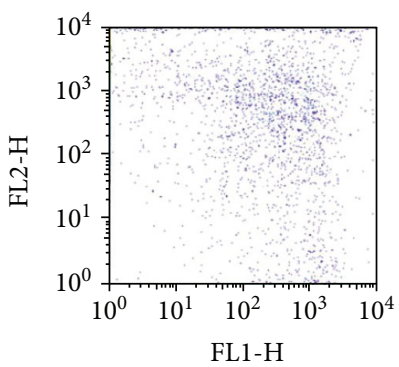

(b)

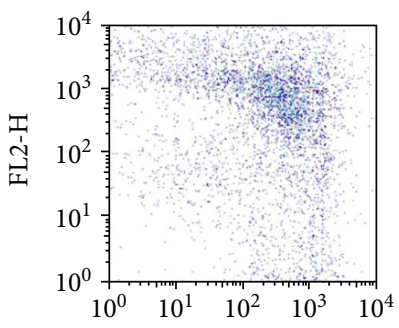

(e)

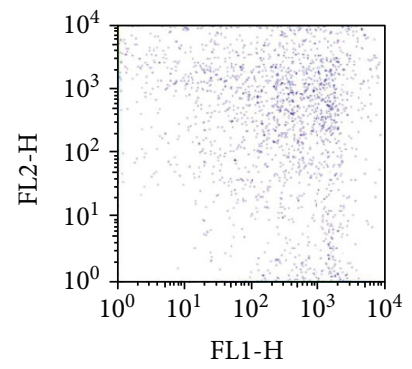

(c)

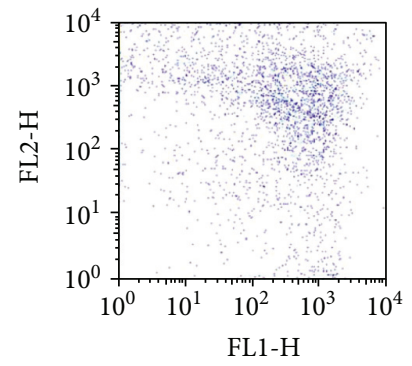

(f)

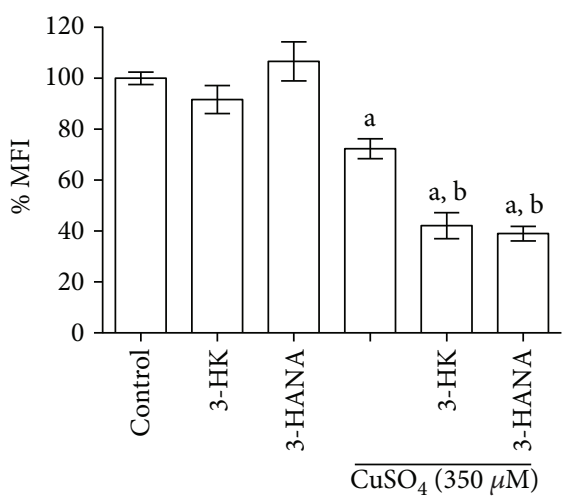

(g)

Figure 5: Effect of coincubation of $\mathrm{CuSO}_{4}(350 \mu \mathrm{M})$ with 3-HK $(100 \mu \mathrm{M})$ and 3-HANA $(100 \mu \mathrm{M})$ on MMP in astrocytes. The MMP was measured using JC-1 orange-red fluorescence. Changes in MMP were evaluated by flow cytometry. Representative dot plots of MMP are showed in (a) control, (b) 3-HK, (c) 3-HANA, (d) copper, (e) copper +3-HK, and (f) copper + 3-HANA. Percentage of mean fluorescence intensity (MFI) is present in (g). Data are presented as mean values + SEM of 6 independent experiments from 3 different cultures. ${ }^{\mathrm{a}} p<0.001$ versus control and ${ }^{\mathrm{b}} p<0.001$ versus $\mathrm{CuSO}_{4}$ (one-way ANOVA followed by Tukey's test).

selective to changes in mitochondrial membrane potential and can form red fluorescence aggregates (FL-1 channel, emission length $525 \mathrm{~nm}$ ) with high MMP, whereas when MMP is low, JC-1 is in its monomeric form (FL-2 channel, emission length at $590 \mathrm{~nm}$ ) and displays a green fluorescence $[50,51]$. After treatments, medium was removed, cells were washed with saline solution, and then $75 \mu \mathrm{l}$ of trypsin was added to each well. Cells were recollected and centrifuged at $2000 \mathrm{rpm}$ for $10 \mathrm{~min}$. Medium was discarded, and mitochondrial membrane potential was evaluated through the label of cells with $3 \mu \mathrm{M}$ of $5,5^{\prime}, 6,6^{\prime}$-tetrachloro-1,1',3,3'-tetraethylbenzimi-dazolylcarbocyanine iodide (JC-1) for $15 \mathrm{~min}$ at $37^{\circ} \mathrm{C}$ in darkness. Then, cells were washed with buffer assay two times. After washing, cells were resuspended and analyzed by flow cytometry. 10,000 events were assessed. Data are expressed as mean fluorescence intensity (MFI) in FL-2 channel and the percentage of cells that decreased MMP [39].

2.6. GSH Determination. GSH concentration was measured with a glutathione detection assay kit (Abcam 65322). Briefly, astrocytes were incubated with copper $(350 \mu \mathrm{M}), 3-\mathrm{HK}$ and 3-HANA $(100 \mu \mathrm{M})$, and with combinations of both in DMEM medium for $24 \mathrm{~h}$ at $37^{\circ} \mathrm{C}$. After that, medium was removed, cells were washed with saline solution, and then $75 \mu \mathrm{l}$ trypsin was added to each well. Cells were recollected $(100,000$ cells $)$ and centrifuged at $2000 \mathrm{rpm}$ for $10 \mathrm{~min}$. Medium was discarded, cells were washed with cold PBS, resuspended in cell lysis buffer, homogenized, and centrifuged $10 \mathrm{~min}$ at $4^{\circ} \mathrm{C}$, and supernatant was collected. The cells were deproteinized with perchloric acid and potassium 


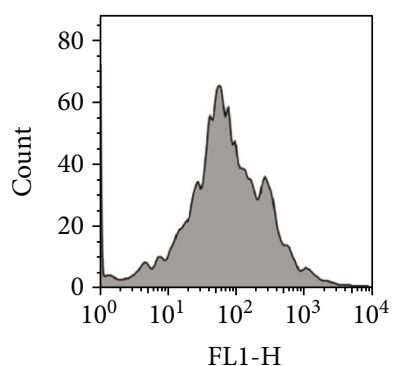

(a)

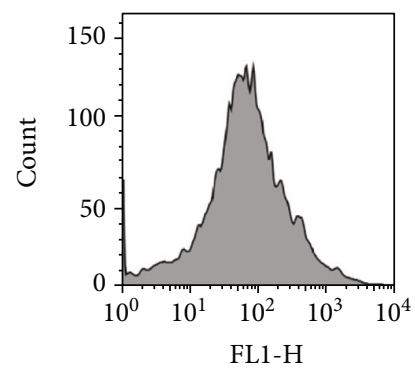

(d)

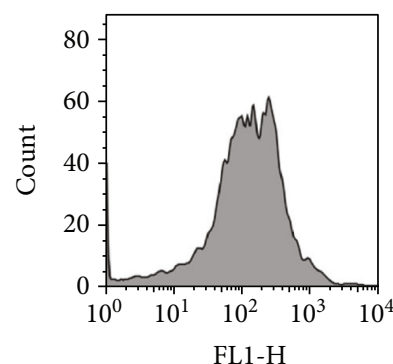

(b)

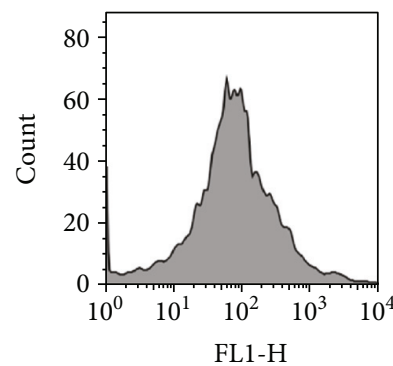

(e)

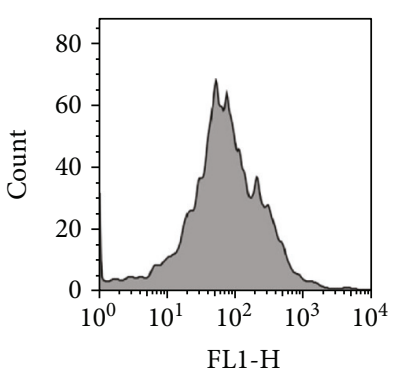

(c)

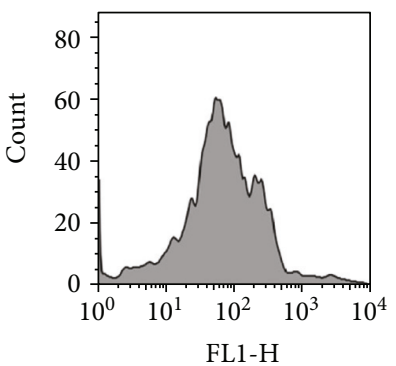

(f)

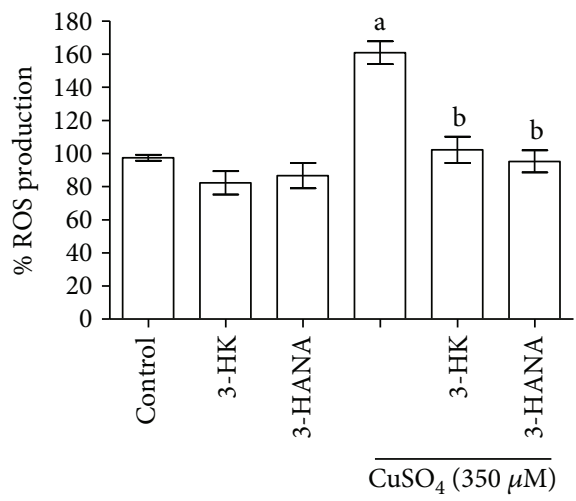

(g)

Figure 6: Effect of 3-HK $(100 \mu \mathrm{M})$ and 3-HANA $(100 \mu \mathrm{M})$ on ROS production induced by copper. After $24 \mathrm{~h}$ of incubation with the kynurenines and copper, DCF-DA was added to all treatments to determinate ROS. Representative dot plots of MMP are showed in (a) control, (b) 3-HK, (c) 3-HANA, (d) copper, (e) copper + 3-HK, and (f) copper + 3-HANA. Percentage of ROS production is showed in (g). Data are presented as mean + SEM of 6 independent experiments from 3 different cultures. ${ }^{\mathrm{a}} p<0.01$ versus control and ${ }^{\mathrm{b}} p<0.001$ versus $\mathrm{CuSO}_{4}$ (one-way ANOVA followed by Tukey's test).

hydroxide. Once deproteinized, samples were ready to use in the GSH determination assay according to the kit's instructions. Briefly, standard curve was prepared from $0.1 \mu \mathrm{g} / \mu \mathrm{l}$ of GSH and dilutions were done in lysis buffer. $50 \mu \mathrm{l}$ of standard and $100 \mu \mathrm{l}$ of each sample were added to each well, and then $2 \mu \mathrm{l}$ of GST reagent and $2 \mu \mathrm{l}$ of monochlorobimane (MCB) were added. The plate was mixed, and fluorescence in samples was immediately measured in a plate reader at $360 \mathrm{~nm}$ excitation and $460 \mathrm{~nm}$ emission in a kinetic mode, every 3 minutes for 1 hour at $37^{\circ} \mathrm{C}$. The results were expressed as the percentage change in glutathione levels in treated versus untreated control samples.

2.7. Viability Test Assay. Cellular death was assessed with propidium iodide according to Magana-Maldonado et al. [52]. Astrocytes were incubated with the combinations of $\mathrm{CuSO}_{4}(350 \mu \mathrm{M})$ with 3 -HK or 3 -HANA $(100 \mu \mathrm{M})$ in
DMEM medium for $24 \mathrm{~h}$ at $37^{\circ} \mathrm{C}$. Then, medium was removed, cells were washed with saline solution, and trypsin $(75 \mu \mathrm{l})$ was added to each well. Cells were recollected and centrifuged at $2000 \mathrm{rpm}$ for $10 \mathrm{~min}$. Medium was discarded, and propidium iodide (PI) was added $(5 \mu \mathrm{g} / \mathrm{ml})$ and incubated for $15 \mathrm{~min}$ in darkness. After incubation with PI, samples were analyzed by flow cytometry, and a total of 10,000 events were assessed. PI fluorescence was determinated with a FACSCalibur instrument, and data collection was performed using unstained cells and positive controls for single color. The results were expressed as cells death percentage.

2.8. Data Analysis. The results were expressed as mean values \pm SEM. All data were analyzed by one-way analysis of variance and Tukey's post hoc test using the Prism software (GraphPad, San Diego, CA, USA). Values of $p<0.05$ were considered statistically significant. 


\section{Results}

3.1. Concentration-Response Effects of Copper Toxicity in Astrocyte Cultures. To evaluate copper toxicity, astrocytes were incubated during $24 \mathrm{~h}$ with different copper concentrations $(0-500 \mu \mathrm{M})$ in DMEM medium. Astrocytes showed decrease on MTT reduction in a concentration-dependent manner in all used concentrations, being the major effect (80\%) with $500 \mu \mathrm{M}$ of copper (Figure 1(a)). After it was proven that copper had effect on cellular function and knowing that the dehydrogenases are responsible of MTT reduction, we evaluated how mitochondrial function was affected and if ROS were implied in copper toxicity on astrocytes. Copper reduced MMP in a concentration-dependent manner (Figure 1(c)) and increased ROS production around $50 \%$ versus control; however, this effect was not concentration dependent (Figure 1(b)). After the toxicity pattern was observed, we evaluated cell death through propidium iodide (PI), which is capable of binding and labeling DNA. After incubation during $24 \mathrm{~h}$ with different concentrations of copper, the cell death increased significantly since the lower concentration $(10 \mathrm{uM})$ was tested and this effect was concentration dependent (Figure 1(d)).

3.2. Copper-Chelating Ability of 3-HK and 3-HANA. With the purpose of testing if copper had an interaction with 3-HK or 3-HANA, we assessed the chelation capacity of 3-HK and 3-HANA for copper. The assay was carried out at three different $\mathrm{pH}(5.5,6.8$, and 7.5) since we knew that copper is able to modify the $\mathrm{pH}$. Figure 2 shows the 3 -HK ability to form a chelating complex with copper in all $\mathrm{pH}$ tested. This 3-HK ability to catch copper was more efficient in acid $\mathrm{pH}$ being that the IC50 is at $\mathrm{pH} 5.5=56.223 \pm 10.322 \mu \mathrm{M}$, IC50 at $\mathrm{pH} 6.8=74.731 \pm 9.3231 \mu \mathrm{M}$, and IC50 at pH $7.5=74.232 \pm 16.769 \mu \mathrm{M}$.

On the other hand, 3-HANA had chelating capacity for copper too, three different conditions were tested, and in all of them, 3-HANA was able to catch copper (Figure 3). At pH 6.8, the most efficient ability to chelate copper with an IC50 $=112.491 \pm 7.212 \mu \mathrm{M}$ took place, following at $\mathrm{pH} 5.5$ with an IC50 $=146.637 \pm 4.922 \mu \mathrm{M}$ and the $\mathrm{pH}$ where it was observed less effect was at $\mathrm{pH} 7.5$ with at IC50 $=559.497 \pm 31.422 \mu \mathrm{M}$.

3.3. Effect of 3-HK and 3-HANA in the Cellular Dysfunction Induced by Copper in Astrocytes. After copper toxicity was evaluated, we determined the kynurenine effect in the presence of this metal $(350 \mu \mathrm{M})$. Figure 4 shows that copper decreases cellular function (around 50\% versus control), evaluated by MTT reduction assay, and the coincubation with the kynurenines enhances this effect (around $80 \%$ versus control). The kynurenines alone do not have effect on MTT reduction.

3.4. Kynurenines Enhance the Reduction of Mitochondrial Membrane Potential Induced by Copper on Astrocytes. The next step was to determine whether the effect on MTT reduction could be related with mitochondrial membrane potential alterations. Representative figures are shown in Figures 5(a), 5(b), 5(c), 5(d), 5(e), and 5(f). 3-HK and 3-HANA (100 $\mu \mathrm{M})$

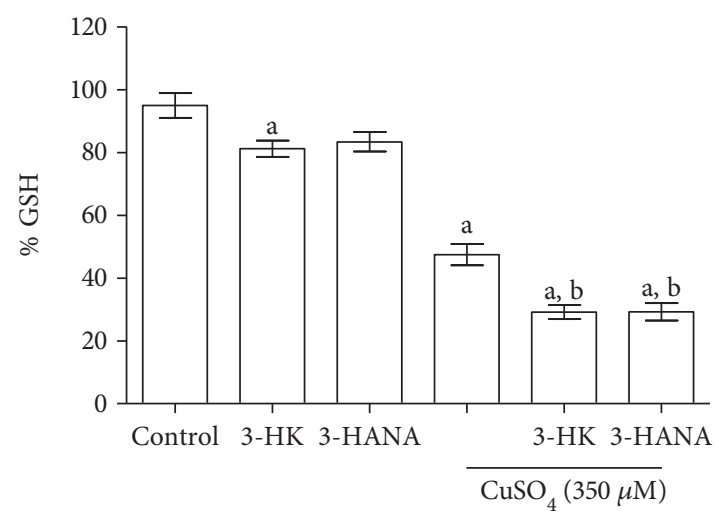

FIGURE 7: Effect of 3-HK and 3-HANA and their coincubation with copper in GSH levels on astrocytes. GSH levels were determinated after $24 \mathrm{~h}$ of incubation with the treatments. Data are presented as mean values + SEM of 8 independent experiments for each treatment. ${ }^{\mathrm{a}} p<0.05$ versus control and ${ }^{\mathrm{b}} p<0.001$ versus $\mathrm{CuSO}_{4}$ (one-way ANOVA followed by Tukey's test).

alone do not induce effect on this parameter compared with the control group. However, copper $(350 \mu \mathrm{M})$ is able to reduce around $40 \%$ the MMP, while the coincubation of copper with both kynurenines reduced around $60 \%$ the MMP versus control.

3.5. 3-HK and 3-HANA Reduce ROS Production Induced by Copper. Considering redox properties of 3-HK and 3-HANA, the next experiment was to know if the potentiation in the copper toxicity induced by the kynurenines was through ROS production. Copper $(350 \mu \mathrm{M})$ induces around $60 \%$ ROS production, and coincubation with $3-\mathrm{HK}$ and 3HANA abolished this effect. Incubation of 3-HK and 3HANA alone did not have effect in ROS production (Figure 6). Representative pictures are shown in Figures 6(a), 6(b), 6(c), and 6(d).

3.6. GSH Depletion Is Involved in Toxicity Pattern Induced by the Coincubation of Copper and Kynurenines. As we know, both copper and kynurenines can interact with GSH, and then we evaluated the levels of this endogenous antioxidant that it is in high concentration in astrocytes. 3-HK decreased GSH levels around 20\%, while copper decreased them around 55\%; 3-HANA did not have effect in this parameter. However, the coincubation of copper with these kynurenines decreased GSH levels around 70\% versus control, in both cases (Figure 7).

3.7. Effect of Copper Coincubation with Kynurenines on Cell Viability. Figure 8 shows the effect of copper and kynurenines on cell viability. Representative pictures are shown in Figures $8(\mathrm{a}), 8(\mathrm{~b}), 8(\mathrm{c}), 8(\mathrm{~d}), 8(\mathrm{e})$, and $8(\mathrm{f})$. 3-HK and 3HANA alone did not have effect on cell death, while copper was able to increase the percentage of dead cells around $45 \%$. Coincubation of copper with kynurenines enhanced the number of dead cells (Figure 8(g)). Figure 9 shows representative bright field micrographs of the different treatments. 3-HK and 3-HANA did not show difference compared with control. However, in copper alone (Figure 9(d)) and the 


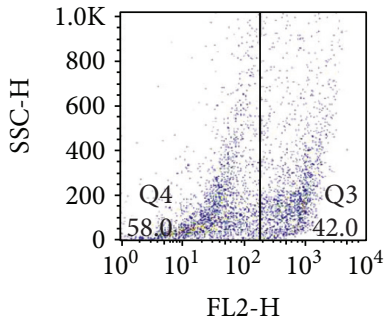

(a)

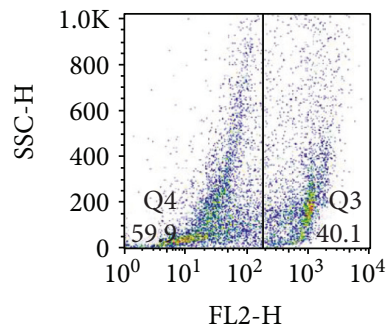

(d)

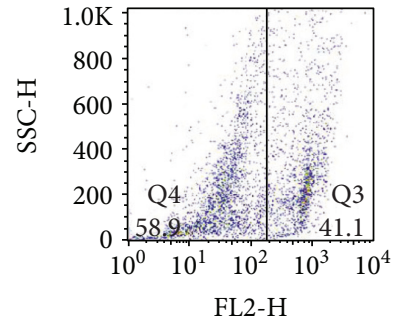

(b)

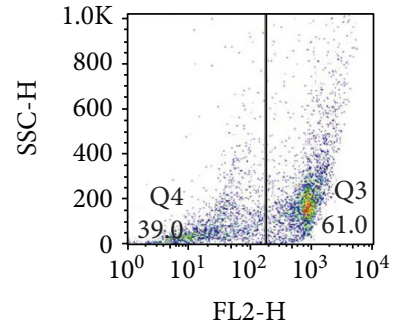

(e)

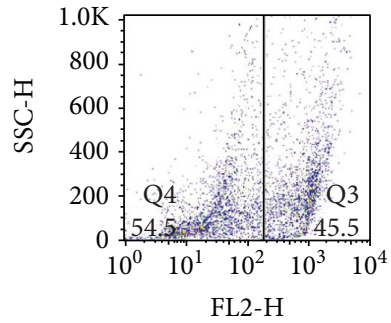

(c)

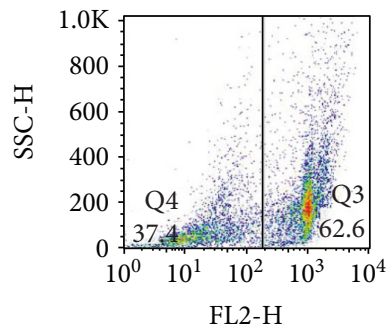

(f)

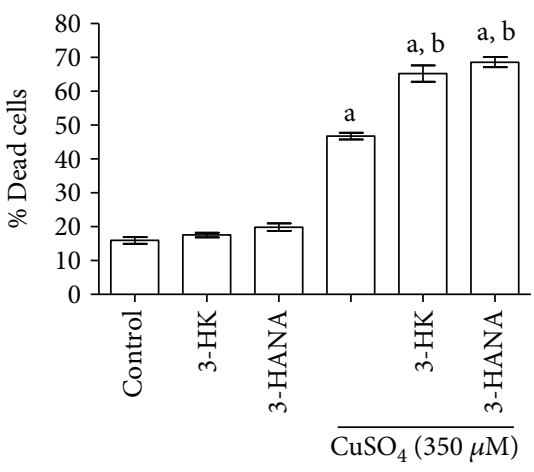

(g)

FIGURE 8: Effect of 3-HK and 3-HANA on cell death induced by copper. The propidium iodide (PI) flow cytometry assay was used for the evaluation of cell viability. Representative dot plots of IP are showed in (a) control, (b) 3-HK, (c) 3-HANA, d) copper, (e) copper + 3-HK, and (f) copper +3 -HANA. Percentage of dead cell is showed in g. Data are presented as mean values + SEM of 6 independent experiments from 3 different cultures. ${ }^{\mathrm{a}} p<0.001$ versus control and ${ }^{\mathrm{b}} p<0.001$ versus $\mathrm{CuSO}_{4}$ (one-way ANOVA followed by Tukey's test).

copper combination with 3-HK (Figure 9(e)) and 3-HANA (Figure 9(f)), a considerable number of dead cells compared with control can be seen.

\section{Discussion}

3-HK and 3-HANA are metabolites of tryptophan catabolism, which possess redox properties and have been associated with neurodegenerative diseases as $\mathrm{HD}$ and $\mathrm{AD}$. These kynurenines are produced through $\mathrm{KP}$, which is highly regulated by redox environment. In this context, copper is an integral part of many important enzymes involved in cellular metabolism; however, its dyshomeostasis can generate oxidative stress and it has been related with some neurodegenerative diseases in which also KP metabolites are involved $[53,54]$. In the present work, we evaluated the effect of $3-\mathrm{HK}$ and 3-HANA in the copper toxicity on astrocytes. We performed the experiments in astrocytes since in the brain, these cells are thought to play a key role in copper homeostasis; in fact, it has been proposed that astrocytes can normally accumulate this metal which will be used by themselves or routed to neurons [53], and the second reason to use astrocytes is that $3-\mathrm{HK}$ and 3-HANA cannot be enzymatically degraded in these cells. First, we demonstrate that copper had toxic effects on astrocytes as some previous reports showed [55-57]. Copper was able to decrease cell functionality and MMP and increase ROS production; these factors may be closely related and be dependent on each other. Despite the fact that copper has important functions as cofactor of antioxidant enzymes as SOD 1, copper is also cytotoxic considering that it can participate in ROS production through Fenton's reaction and to displace other elemental metals [58, 59]. Besides, mitochondrial alterations and changes in redox environment induced by copper can lead to decrease cell viability as was observed in this work.

On the other hand, it has been shown that 3-HK and 3HANA are able to scavenge hydroxyl radical and peroxynitrite in chemical combinatory assays and also are able to chelate some metals such as iron [39]. Keeping in mind this background, we explore if 3 -HK and 3-HANA would have 


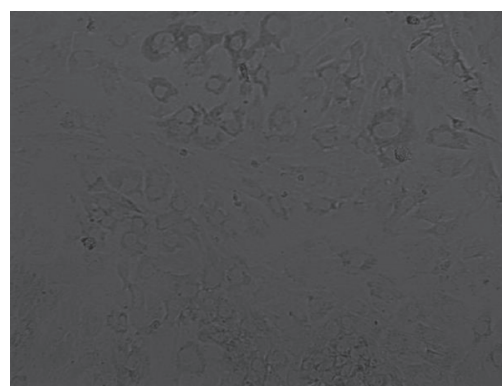

(a)

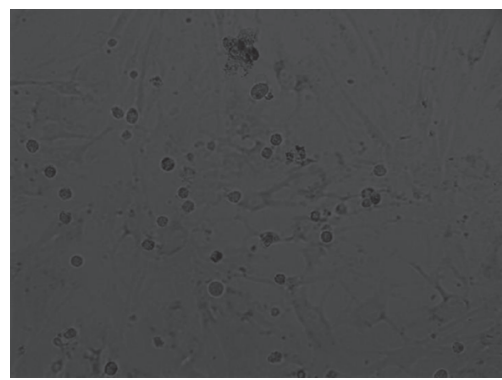

(d)

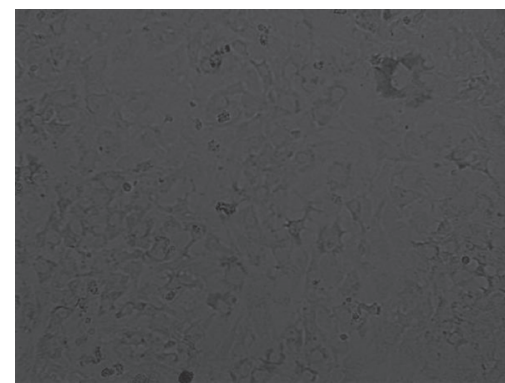

(b)

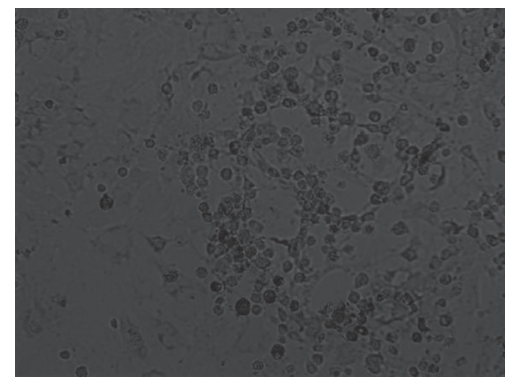

(e)

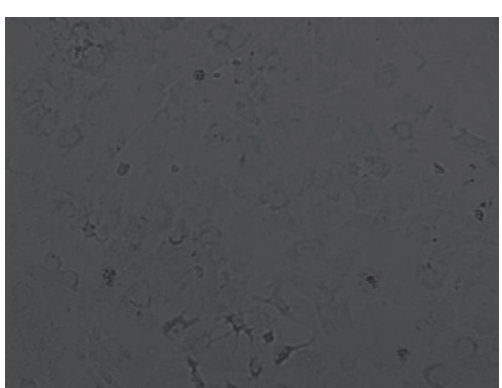

(c)

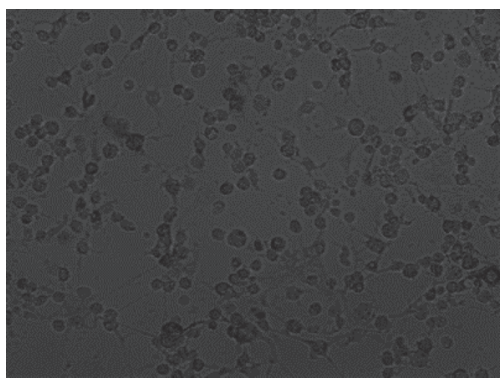

(f)

Figure 9: Effect of kynurenines on cell death induced by copper in astrocytes. Representative phase-contrast micrographs showing the effect of coincubation of 3-HK or 3-HANA with copper. (a) Control, (b) $3-\mathrm{HK}$, (c) 3-HANA, (d) $\mathrm{CuSO}_{4},(\mathrm{e}) \mathrm{CuSO}_{4}+3-\mathrm{HK}^{-}$ and (f) $\mathrm{CuSO}_{4}+3$-HANA.

copper-chelating capability. Copper-chelating probes were placed at three different $\mathrm{pH}(5.5,6.8$, and 7.5$)$ by two reasons: (1) in the method that we are using to determinate copper chelation, the affinity of hematoxylin for cupric ions is decreased when the $\mathrm{pH}$ is also reduced, and mainly (2) because it has been described in previous reports that copper accumulation, as in some pathologies, can change environment $\mathrm{pH}$ and this changes can influence the chelating capacity of various molecules [44-46]. Our data show that both kynurenine metabolites were able to chelate copper under different conditions of $\mathrm{pH}$; this results may be due to the nature of 3-HK and 3-HANA since it has been reported that these metabolites are good electron donors in electrochemical experiments [60].

According to redox and chelating properties of 3-HK and 3-HANA, we decided to evaluate their effect on the copper toxicity. Both kynurenines were able to abolish ROS production induced by copper; however, the toxic effect on mitochondrial and cellular function was enhanced by the coincubation of copper with both kynurenines. These effects may be due to the fact that both 3-HK and 3-HANA are able to affect respiratory control (oxygen consumption in states 2 and 3 of mitochondrial respiration) [61] in addition to copper toxicity in mitochondria. Both sceneries affect ATP production and can lead to cell death as can be observed in Figure 9. In this context, a previous report showed that 3HK and 3-HANA produced cellular damage but in an independent way of ROS production, and actually, the oxidative stress parameters evaluated were even below of basal levels [39]. Moreover, the toxic copper effect enhanced by the kynurenines can be due to the fact that these kynurenines can be oxidized in the presence of copper as copper is reduced, promoting cross-linking in important proteins [34]. Besides, autoxidation of these hydroxykynurenines can form compounds as xanthommatin radical, p-quinone, and 4,6dihydroxyquinolinequinonecarboxylic acid (DHQCA) which are reactive [62] and can interfere with the mitochondrial function and subsequently provoke cell toxicity. Other parameter evaluated was the effect of copper and kynurenines on GSH, which is in high amounts in astrocytes [17]. GSH is responsible to form complexes with copper being copper a natural pool in astrocytes; this complex is required for the incorporation of copper into metallothionein and for SOD activation. In the case of copper was overmuch, GSH would be the first antioxidant to catch it and avoid a triggering of oxidative effects [63-65]. Our data show that copper $(350 \mu \mathrm{M})$ decreased GSH levels and this effect was also enhanced by 3-HK and 3-HANA. This could be explained by the fact that in astrocytes, 3-HK can suffer a deamination and this could occur slowly at physiological $\mathrm{pH}$ or by the action of kynurenine aminotransferase (KAT), forming 3-hydroxykynurenine glucoside (3-OHKG) in a nonoxidative way, which in turn can form adducts with GSH [66-68]. With the knowledge that KAT is the most abundant KP enzyme in astrocytes, it is not hard to think that 3-HK deamination is taking place and 3-OHKG can form adducts with the large amounts of GSH in this cell type, decreasing GSH available to catch copper and allowing that free copper causes the greater damage observed. Although that process has not been described with 3-HANA, it has been shown that this metabolite is also able to decrease GSH levels in some kind of cells [69]. It is important to take into consideration that 3-HK and 3-HANA could be good ROS scavengers and chelating 
agents; however, their interaction with other cellular components could increase the cell vulnerability to damage toward other agents, as in this case, to copper.

\section{Conclusion}

This research provides important evidence about how two endogen KP metabolites can intensify the cellular damage induced by copper. It is relevant, because in some neurodegenerative diseases, they are found as common factors, the alteration in copper concentrations or in other metals, as well as KP metabolite alterations. The challenge for the future research would be to know the precise modulation of $\mathrm{KP}$ metabolites by metals and try to identify therapeutic targets in diseases where these components are present.

\section{Conflicts of Interest}

The authors report no conflicts of interest.

\section{Authors' Contributions}

The authors alone are responsible for the content and writing of the paper.

\section{Acknowledgments}

This work was supported by CONACYT Grant 262010.

\section{References}

[1] A. Ferrer, "Metal poisoning," Anales del Sistema Sanitario de Navarra, vol. 26, Supplement 1, pp. 141-153, 2003.

[2] M. Valko, K. Jomova, C. J. Rhodes, K. Kuca, and K. Musílek, "Redox- and non-redox-metal-induced formation of free radicals and their role in human disease," Archives of Toxicology, vol. 90, no. 1, pp. 1-37, 2016.

[3] M. Valko, H. Morris, and M. T. Cronin, "Metals, toxicity and oxidative stress," Current Medicinal Chemistry, vol. 12, no. 10, pp. 1161-1208, 2005.

[4] L. Lewinska-Preis, M. Jabłońska, M. J. Fabiańska, and A. Kita, "Bioelements and mineral matter in human livers from the highly industrialized region of the Upper Silesia Coal Basin (Poland)," Environmental Geochemistry and Health, vol. 33, no. 6, pp. 595-611, 2011.

[5] A. I. Bush, "Metals and neuroscience," Current Opinion in Chemical Biology, vol. 4, no. 2, pp. 184-191, 2000.

[6] S. Ferguson-Miller and G. T. Babcock, "Heme/copper terminal oxidases," Chemical Reviews, vol. 96, no. 7, pp. 2889-2908, 1996.

[7] D. M. Popovic, I. V. Leontyev, D. G. Beech, and A. A. Stuchebrukhov, "Similarity of cytochrome c oxidases in different organisms," Proteins, vol. 78, no. 12, pp. 2691-2698, 2010.

[8] J. P. Klinman, "The copper-enzyme family of dopamine betamonooxygenase and peptidylglycine alpha-hydroxylating monooxygenase: resolving the chemical pathway for substrate hydroxylation," The Journal of Biological Chemistry, vol. 281, no. 6, pp. 3013-3016, 2006.

[9] F. Perez-Aguilar, "Ceruloplasmine and iron metabolism: their implications in hemochromatosis, Wilson's disease and aceruloplasminemia," Revista Clínica Española, vol. 202, no. 12, pp. 649-651, 2002.

[10] J. Healy and K. Tipton, "Ceruloplasmin and what it might do," Journal Neural Transmission (Vienna), vol. 114, no. 6, pp. 777-781, 2007.

[11] M. M. Pena, J. Lee, and D. J. Thiele, "A delicate balance: homeostatic control of copper uptake and distribution," The Journal of Nutrition, vol. 129, no. 7, pp. 1251-1260, 1999.

[12] P. N. Lanken, "The challenge of medical decision making. Balancing patient autonomy and physician responsibility," The American Review of Respiratory Disease, vol. 145, no. 2, Part 1, pp. 253-254, 1992.

[13] J. R. Burdo, S. L. Menzies, I. A. Simpson et al., "Distribution of divalent metal transporter 1 and metal transport protein 1 in the normal and Belgrade rat," Journal of Neuroscience Research, vol. 66, no. 6, pp. 1198-1207, 2001.

[14] J. F. Eisses and J. H. Kaplan, "Molecular characterization of hCTR1, the human copper uptake protein," The Journal of Biological Chemistry, vol. 277, no. 32, pp. 29162-29171, 2002.

[15] S. A. Lee, O. V. Belyaeva, and N. Y. Kedishvili, "Biochemical characterization of human epidermal retinol dehydrogenase 2," Chemico-Biological Interactions, vol. 178, no. 1-3, pp. 182-187, 2009.

[16] M. Aschner, "Astrocyte metallothioneins (MTs) and their neuroprotective role," Annals of the New York Academy of Sciences, vol. 825, pp. 334-347, 1997.

[17] J. Hirrlinger and R. Dringen, "The cytosolic redox state of astrocytes: maintenance, regulation and functional implications for metabolite trafficking," Brain Research Reviews, vol. 63, no. 1-2, pp. 177-188, 2010.

[18] H. Kodama, C. Fujisawa, and W. Bhadhprasit, "Pathology, clinical features and treatments of congenital copper metabolic disorders-focus on neurologic aspects," Brain Development, vol. 33, no. 3, pp. 243-251, 2011.

[19] E. Gaggelli, H. Kozlowski, D. Valensin, and G. Valensin, "Copper homeostasis and neurodegenerative disorders (Alzheimer's, prion, and Parkinson's diseases and amyotrophic lateral sclerosis)," Chemical Reviews, vol. 106, no. 6, pp. 1995-2044, 2006.

[20] M. A. Greenough, J. Camakaris, and A. I. Bush, "Metal dyshomeostasis and oxidative stress in Alzheimer's disease," Neurochemistry International, vol. 62, no. 5, pp. 540-555, 2013.

[21] R. Meamar, H. Nikyar, L. Dehghani, K. Basiri, and M. R. Ghazvini, "Assessing of plasma levels of iron, zinc and copper in Iranian Parkinson's disease," Advanced Biomedical Research, vol. 5, p. 31, 2016.

[22] G. Allegri, G. Allegri, C. V. Costa, A. Bertazzo, M. Biasiolo, and E. Ragazzi, "Enzyme activities of tryptophan metabolism along the kynurenine pathway in various species of animals," Farmaco, vol. 58, no. 9, pp. 829-836, 2003.

[23] C. A. Opitz and I. Heiland, "Dynamics of NAD-metabolism: everything but constant," Biochemical Society Transactions, vol. 43, no. 6, pp. 1127-1132, 2015.

[24] G. Allegri, "Effects of two different loading doses of Ltryptophan on the urinary excretion of tryptophan metabolites in rats, mice and guinea pigs. Correlation with the enzyme activities," The Italian Journal of Biochemistry, vol. 36, no. 3, pp. 194-203, 1987.

[25] G. Allegri, E. Ragazzi, A. Bertazzo, M. Biasiolo, and C. V. Costa, "Tryptophan metabolism in rabbits," Advances in Experimental Medicine and Biology, vol. 527, pp. 473-479, 2003. 
[26] J. Reyes Ocampo, R. Lugo Huitrón, D. González-Esquivel et al., "Kynurenines with neuroactive and redox properties: relevance to aging and brain diseases," Oxidative Medicine and Cellular Longevity, vol. 2014, Article ID 646909, 22 pages, 2014.

[27] R. Schwarcz, "The kynurenine pathway of tryptophan degradation as a drug target," Current Opinion in Pharmacology, vol. 4, no. 1, pp. 12-17, 2004.

[28] T. R. Guilarte and C. L. Eastman, "Is 3-hydroxykynurenine an endogenous neurotoxin in Huntington's disease?" Journal of the Neurological Sciences, vol. 116, no. 2, pp. 227-228, 1993.

[29] S. J. Pearson and G. P. Reynolds, "Increased brain concentrations of a neurotoxin, 3-hydroxykynurenine, in Huntington's disease," Neuroscience Letters, vol. 144, no. 1-2, pp. 199-201, 1992.

[30] C. L. Eastman and T. R. Guilarte, "Cytotoxicity of 3hydroxykynurenine in a neuronal hybrid cell line," Brain Research, vol. 495, no. 2, pp. 225-231, 1989.

[31] Y. Nakagami, H. Saito, and H. Katsuki, "3-Hydroxykynurenine toxicity on the rat striatum in vivo," Japanese Journal of Pharmacology, vol. 71, no. 2, pp. 183-186, 1996.

[32] S. Okuda, N. Nishiyama, H. Saito, and H. Katsuki, "Hydrogen peroxide-mediated neuronal cell death induced by an endogenous neurotoxin, 3-hydroxykynurenine," Proceedings of the National Academy of Sciences of the United States of America, vol. 93, no. 22, pp. 12553-12558, 1996.

[33] S. Okuda, N. Nishiyama, H. Saito, and H. Katsuki, "3-Hydroxykynurenine, an endogenous oxidative stress generator, causes neuronal cell death with apoptotic features and region selectivity," Journal of Neurochemistry, vol. 70, no. 1, pp. 299-307, 1998.

[34] L. E. Goldstein, M. C. Leopold, X. Huang et al., "3-Hydroxykynurenine and 3-hydroxyanthranilic acid generate hydrogen peroxide and promote alpha-crystallin cross-linking by metal ion reduction," Biochemistry, vol. 39, no. 24, pp. 7266-7275, 2000.

[35] S. Vazquez, J. A. Aquilina, J. F. Jamie, M. M. Sheil, and R. J. Truscott, "Novel protein modification by kynurenine in human lenses," The Journal of Biological Chemistry, vol. 277, no. 7, pp. 4867-4873, 2002.

[36] G. Leipnitz, G. Leipnitz, C. Schumacher et al., "In vitro evidence for an antioxidant role of 3-hydroxykynurenine and 3-hydroxyanthranilic acid in the brain," Neurochemistry International, vol. 50, no. 1, pp. 83-94, 2007.

[37] N. Goshima, A. Wadano, and K. Miura, "3-Hydroxykynurenine as O2-. Scavenger in the blowfly, Aldrichina grahami," Biochemical and Biophysical Research Communications, vol. 139, no. 2, pp. 666-672, 1986.

[38] C. Backhaus, H. Rahman, S. Scheffler, H. Laatsch, and R. Hardeland, "NO scavenging by 3-hydroxyanthranilic acid and 3-hydroxykynurenine: $\mathrm{N}$-nitrosation leads via oxadiazoles to o-quinone diazides," Nitric Oxide, vol. 19, no. 3, pp. 237244, 2008.

[39] J. Reyes-Ocampo, D. Ramírez-Ortega, G. V. Cervantes et al., "Mitochondrial dysfunction related to cell damage induced by 3-hydroxykynurenine and 3-hydroxyanthranilic acid: non-dependent-effect of early reactive oxygen species production," Neurotoxicology, vol. 50, pp. 81-91, 2015.

[40] E. Quagliariello, F. Palmieri, A. Alifano, and S. Papa, "3Hydroxyanthranilic acid-mediated respiration in the inhibited respiratory chain," Biochimica et Biophysica Acta (BBA) Enzymology and Biological Oxidation, vol. 113, no. 3, pp. 482-489, 1966.
[41] E. Quagliariello, S. Papa, C. Saccone, and A. Alifano, "Effect of 3-hydroxyanthranilic acid on the mitochondrial respiratory system," The Biochemical Journal, vol. 91, no. 1, pp. 137-146, 1964.

[42] N. Braidy, R. Grant, B. J. Brew, S. Adams, T. Jayasena, and G. J. Guillemin, "Effects of kynurenine pathway metabolites on intracellular $\mathrm{NAD}^{+}$synthesis and cell death in human primary astrocytes and neurons," International Journal of Tryptophan Research, vol. 2, pp. 61-69, 2009.

[43] D. Krause, H. S. Suh, L. Tarassishin et al., "The tryptophan metabolite 3-hydroxyanthranilic acid plays antiinflammatory and neuroprotective roles during inflammation: role of hemeoxygenase-1," The American Journal of Pathology, vol. 179, no. 3, pp. 1360-1372, 2011.

[44] M. Riha, J. Karlíčková, T. Filipský, K. Macáková, R. Hrdina, and P. Mladěnka, "Novel method for rapid copper chelation assessment confirmed low affinity of D-penicillamine for copper in comparison with trientine and 8-hydroxyquinolines," Journal of Inorganic Biochemistry, vol. 123, pp. 8087, 2013.

[45] J. F. Collins, J. R. Prohaska, and M. D. Knutson, "Metabolic crossroads of iron and copper," Nutrition Reviews, vol. 68, no. 3, pp. 133-147, 2010.

[46] A. Yagi, H. Hayashi, T. Higuchi, N. Hishida, and N. Sakamoto, "Three stages of copper accumulation in hepatocellular lysosomes: X-ray microanalysis of copper-loaded golden hamsters," International Journal of Experimental Pathology, vol. 73, no. 1, pp. 85-94, 1992.

[47] V. Pérez-De La Cruz, D. Elinos-Calderón, P. Carrillo-Mora et al., "Time-course correlation of early toxic events in three models of striatal damage: modulation by proteases inhibition," Neurochemistry International, vol. 56, no. 6-7, pp. 834-842, 2010.

[48] M. N. Herrera-Mundo, D. Silva-Adaya, P. D. Maldonado et al., "S-Allylcysteine prevents the rat from 3-nitropropionic acidinduced hyperactivity, early markers of oxidative stress and mitochondrial dysfunction," Neuroscience Research, vol. 56, no. 1, pp. 39-44, 2006.

[49] R. Lugo-Huitron, T. Blanco-Ayala, P. Ugalde-Muniz et al., "On the antioxidant properties of kynurenic acid: free radical scavenging activity and inhibition of oxidative stress," Neurotoxicology and Teratology, vol. 33, no. 5, pp. 538-547, 2011.

[50] D. R. Green and J. C. Reed, "Mitochondria and apoptosis," Science, vol. 281, no. 5381, pp. 1309-1312, 1998.

[51] S. Salvioli, A. Ardizzoni, C. Franceschi, and A. Cossarizza, "JC1 , but not $\operatorname{DiOC}_{6}(3)$ or rhodamine 123 , is a reliable fluorescent probe to assess $\Delta \Psi$ changes in intact cells: implications for studies on mitochondrial functionality during apoptosis," FEBS Letters, vol. 411, no. 1, pp. 77-82, 1997.

[52] R. Magana-Maldonado, K. Manoutcharian, N. Y. HernándezPedro et al., "Concomitant treatment with pertussis toxin plus temozolomide increases the survival of rats bearing intracerebral RG2 glioma," Journal of Cancer Research and Clinical Oncology, vol. 140, no. 2, pp. 291-301, 2014.

[53] B. Sullivan, G. Robison, Y. Pushkar, J. K. Young, and K. F. Manaye, "Copper accumulation in rodent brain astrocytes: a species difference," Journal of Trace Elements in Medicine and Biology, vol. 39, pp. 6-13, 2017.

[54] S. Manoharan, G. J. Guillemin, R. S. Abiramasundari, M. M. Essa, M. Akbar, and M. D. Akbar, "The role of reactive oxygen species in the pathogenesis of Alzheimer's disease, 
Parkinson's disease, and Huntington's disease: a mini review," Oxidative Medicine and Cellular Longevity, vol. 2016, Article ID 8590578, 15 pages, 2016.

[55] H. L. Hu, X. S. Ni, S. Duff-Canning, and X. P. Wang, "Oxidative damage of copper chloride overload to the cultured rat astrocytes," American Journal of Translational Research, vol. 8, no. 2, pp. 1273-1280, 2016.

[56] F. Bulcke, P. Santofimia-Castaño, A. Gonzalez-Mateos, and R. Dringen, "Modulation of copper accumulation and copper-induced toxicity by antioxidants and copper chelators in cultured primary brain astrocytes," Journal of Trace Elements in Medicine and Biology, vol. 32, pp. 168-176, 2015.

[57] P. V. Reddy, K. V. Rao, and M. D. Norenberg, "The mitochondrial permeability transition, and oxidative and nitrosative stress in the mechanism of copper toxicity in cultured neurons and astrocytes," Laboratory Investigation, vol. 88, no. 8, pp. 816-830, 2008.

[58] S. Ferruzza, S. Ferruzza, Y. Sambuy et al., "Copper uptake and intracellular distribution in the human intestinal Caco-2 cell line," Biometals, vol. 13, no. 2, pp. 179-185, 2000.

[59] A. V. Gyulkhandanyan, C. J. Feeney, and P. S. Pennefather, "Modulation of mitochondrial membrane potential and reactive oxygen species production by copper in astrocytes," Journal of Neurochemistry, vol. 87, no. 2, pp. 448460, 2003.

[60] G. I. Giles, C. A. Collins, T. W. Stone, and C. Jacob, "Electrochemical and in vitro evaluation of the redox-properties of kynurenine species," Biochemical and Biophysical Research Communications, vol. 300, no. 3, pp. 719-724, 2003.

[61] H. Baran, K. Staniek, M. Bertignol-Spörr, M. Attam, C. Kronsteiner, and B. Kepplinger, "Effects of various kynurenine metabolites on respiratory parameters of rat brain, liver and heart mitochondria," International Journal of Tryptophan Research, vol. 9, pp. 17-29, 2016.

[62] S. Vazquez, B. Garner, M. M. Sheil, and R. J. Truscott, "Characterisation of the major autoxidation products of 3hydroxykynurenine under physiological conditions," Free Radical Research, vol. 32, no. 1, pp. 11-23, 2000.

[63] I. Ascone, A. Longo, H. Dexpert, M. R. Ciriolo, G. Rotilio, and A. Desideri, "An X-ray absorption study of the reconstitution process of bovine $\mathrm{Cu}, \mathrm{Zn}$ superoxide dismutase by $\mathrm{Cu}(\mathrm{I})$-glutathione complex," FEBS Letters, vol. 322, no. 2, pp. 165-167, 1993.

[64] M. R. Ciriolo, A. Desideri, M. Paci, and G. Rotilio, "Reconstitution of $\mathrm{Cu}, \mathrm{Zn}$-superoxide dismutase by the $\mathrm{Cu}(\mathrm{I})$.glutathione complex," The Journal of Biological Chemistry, vol. 265, no. 19, pp. 11030-11034, 1990.

[65] A. M. Ferreira, A. D. Ferreira, M. R. Ciriolo, L. Marcocci, and G. Rotilio, "Copper(I) transfer into metallothionein mediated by glutathione," The Biochemical Journal, vol. 292, Part 3, pp. 673-676, 1993.

[66] R. Van Heyningen, "Fluorescent glucoside in the human lens," Nature, vol. 230, no. 5293, pp. 393-394, 1971.

[67] B. Garner, S. Vazquez, R. Griffith, R. A. Lindner, J. A. Carver, and R. J. Truscott, "Identification of glutathionyl3-hydroxykynurenine glucoside as a novel fluorophore associated with aging of the human lens," The Journal of Biological Chemistry, vol. 274, no. 30, pp. 20847-20854, 1999.
[68] B. Garner, B. Garner, D. C. Shaw, R. A. Lindner, J. A. Carver, and R. J. Truscott, "Non-oxidative modification of lens crystallins by kynurenine: a novel post-translational protein modification with possible relevance to ageing and cataract," Biochimica et Biophysica Acta (BBA) - Protein Structure and Molecular Enzymology, vol. 1476, no. 2, pp. 265-278, 2000.

[69] S. M. Lee, Y. S. Lee, J. H. Choi et al., "Tryptophan metabolite 3hydroxyanthranilic acid selectively induces activated $\mathrm{T}$ cell death via intracellular GSH depletion," Immunology Letters, vol. 132, no. 1-2, pp. 53-60, 2010. 


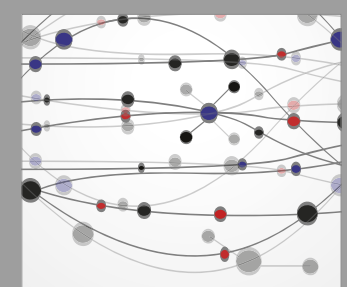

The Scientific World Journal
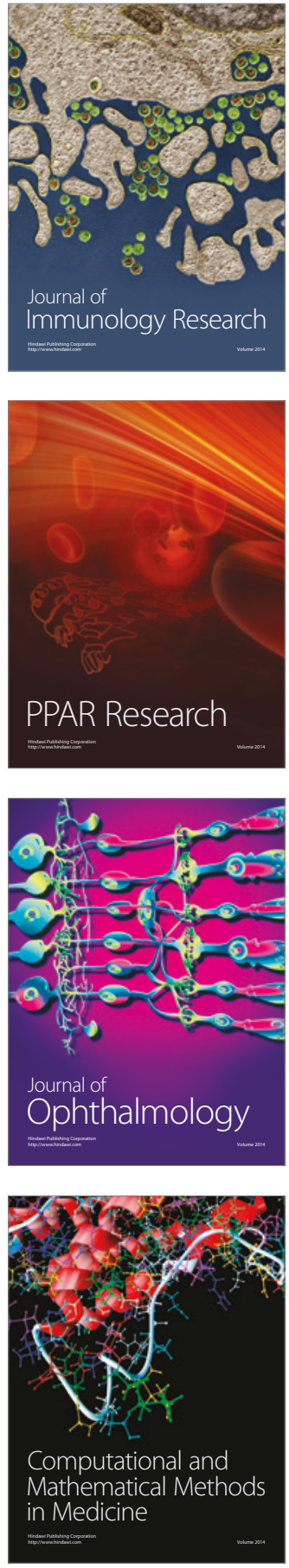

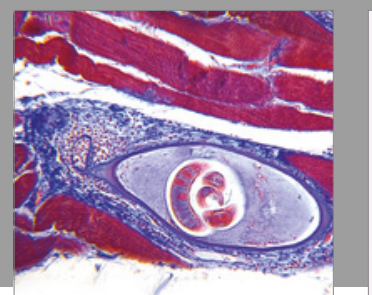

Gastroenterology Research and Practice
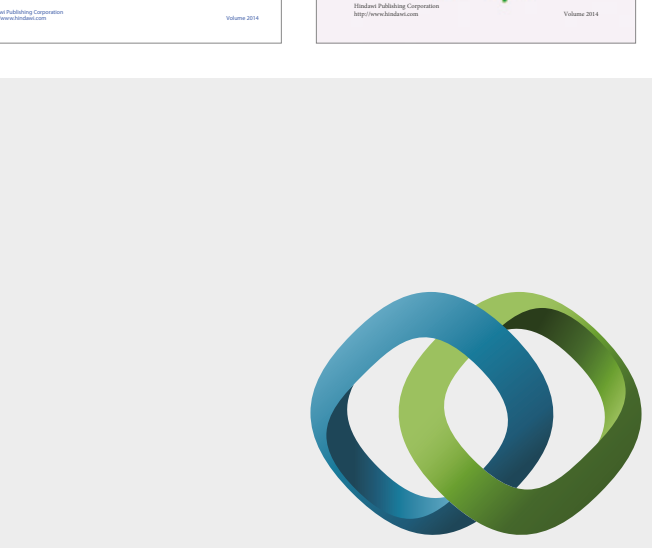

\section{Hindawi}

Submit your manuscripts at

https://www.hindawi.com
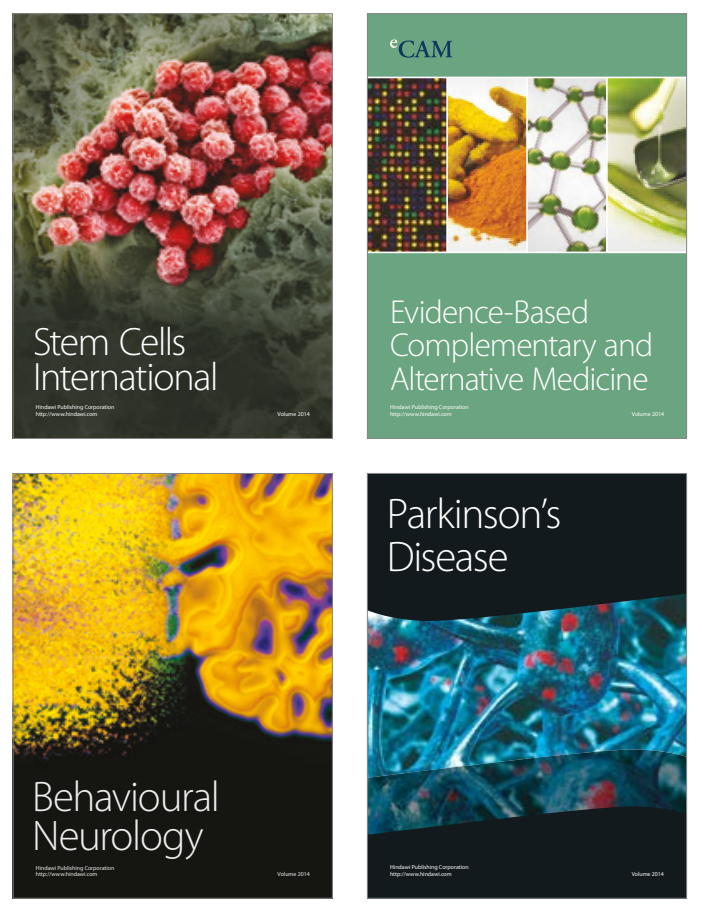
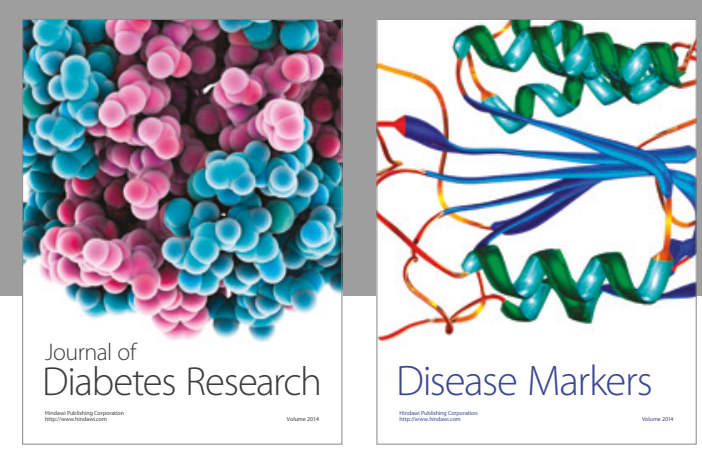

Disease Markers
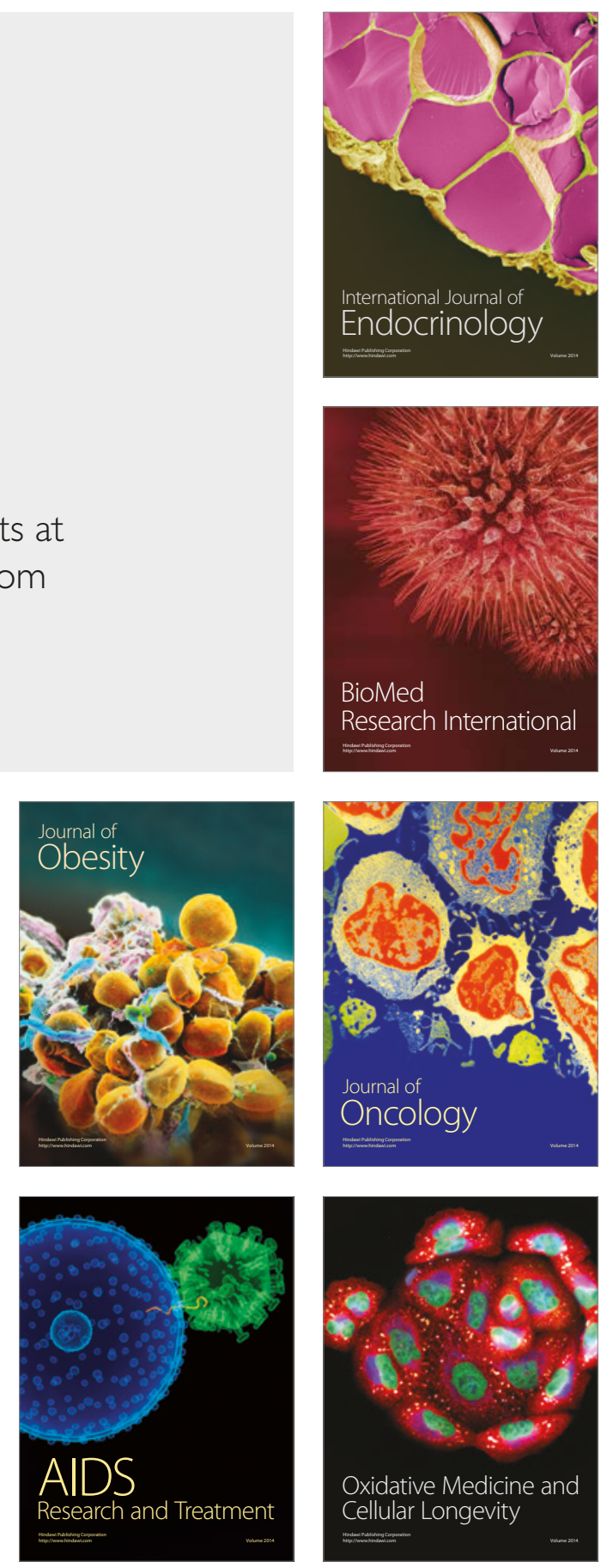